\title{
A Pieri rule for skew shapes
}

\author{
Sami H. Assaf 1 tIand Peter R. W. McNamara ${ }^{2}$ \\ ${ }^{1}$ Department of Mathematics, Massachusetts Institute of Technology, 77 Massachusetts Avenue, Cambridge, MA \\ 02139, USA \\ ${ }^{2}$ Department of Mathematics, Bucknell University, Lewisburg, PA 17837, USA \\ sassaf@math.mit.edu, peter.mcnamara@bucknell.edu
}

\begin{abstract}
The Pieri rule expresses the product of a Schur function and a single row Schur function in terms of Schur functions. We extend the classical Pieri rule by expressing the product of a skew Schur function and a single row Schur function in terms of skew Schur functions. Like the classical rule, our rule involves simple additions of boxes to the original skew shape. Our proof is purely combinatorial and extends the combinatorial proof of the classical case.

Résumé. La règle de Pieri exprime le produit d'une fonction de Schur et de la fonction de Schur d'une seule ligne en termes de fonctions de Schur. Nous étendons la règle classique de Pieri en exprimant le produit d'un fonction gauche de Schur et de la fonction de Schur d'une ligne en termes de fonctions gauches de Schur. Comme la règle classique, notre règle implique l'ajout de cases à la forme gauche initiale. Notre preuve est purement combinatoire et étend celle du cas classique.
\end{abstract}

Keywords: Pieri rule, skew Schur functions, Robinson-Schensted

\section{Introduction}

The basis of Schur functions is arguably the most interesting and important basis for the ring of symmetric functions. This is due not just to their elegant combinatorial definition, but more broadly to their connections to other areas of mathematics. For example, they are intimately tied to the cohomology ring of the Grassmannian, and they appear in the representation theory of the symmetric group and of the general and special linear groups.

It is therefore natural to consider the expansion of the product $s_{\lambda} s_{\mu}$ of two Schur functions in the basis of Schur functions. The Littlewood-Richardson rule [LR34, Sch77, Tho74, Tho78], which now comes in many different forms ([Sta99] is one starting point), allows us to determine this expansion. However, more basic than the Littlewood-Richardson rule is the Pieri rule, which gives a simple, beautiful and more intuitive answer for the special case when $\mu=(n)$, a partition of length 1 . Though we will postpone the preliminary definitions to Section 2 and the statement of the Pieri rule to Section 3 , stating the rule in a rough form will give its flavor. For a partition $\lambda$ and a positive integer $n$, the Pieri rule states that

\footnotetext{
†Supported by NSF Postdoctoral Fellowship DMS-0703567

1365-8050 (c) 2005 Discrete Mathematics and Theoretical Computer Science (DMTCS), Nancy, France
} 
$s_{\lambda} s_{n}$ is a sum of Schur functions $s_{\lambda^{+}}$, where $\lambda^{+}$is obtainable by adding cells to the diagram of $\lambda$ according to a certain simple rule. The Pieri rule's prevalence is highlighted by its adaptions to many other settings, including Schubert polynomials [LS82, LS07, Man98, Sot96, Win98], Hall-Littlewood polynomials [Mor64], Jack polynomials [Las89, Sta89], LLT polynomials [Lam05], and Macdonald polynomials [Koo88, Mac87, Mac95].

It is therefore surprising that there does not appear to be a known adaption of the Pieri rule to the most well-known generalization of Schur functions, namely skew Schur functions. We fill this gap in the literature with a natural extension of the Pieri rule to the skew setting. Reflecting the simplicity of the classical Pieri rule, the skew Pieri rule states that for a skew shape $\lambda / \mu$ and a positive integer $n, s_{\lambda / \mu} s_{n}$ is a signed sum of skew Schur functions $s_{\lambda^{+} / \mu^{-}}$, where $\lambda^{+} / \mu^{-}$is obtainable by adding cells to the diagram of $\lambda / \mu$ according to a certain simple rule. Our proof is purely combinatorial, using a sign-reversing involution that reflects the combinatorial proof of the classical Pieri rule.

After reading an early version of the full version [AM09] of this manuscript, which included an algebraic proof of the case $n=1$ due to Richard Stanley, Thomas Lam provided a complete algebraic proof of our skew Pieri rule, which is included as an appendix to [AM09]. It is natural to ask if our skew Pieri rule can be extended to give a "skew" version of the Littlewood-Richardson rule, and we included such a rule as a conjecture in [AM09]. This conjecture has been proved by Lam, Aaron Lauve and Frank Sottile in [LLS09] using Hopf algebras. It remains an open problem to find a combinatorial proof of the skew Littlewood-Richardson rule.

The remainder of this paper is organized as follows. In Section 2, we give the necessary symmetric function background. In Section 3, we state the classical Pieri rule and introduce our skew Pieri rule. In Section 4, we give a variation from [SS90] of the Robinson-Schensted-Knuth algorithm, along with relevant properties. This algorithm is then used in Section 5 to define the sign-reversing involution that proves the skew Pieri rule.

\subsection{Acknowledgments}

We are grateful to a number of experts for informing us that they too were surprised by the existence of the skew Pieri rule, and particularly to Richard Stanley for providing an algebraic proof of the $n=1$ case that preceded our combinatorial proof. This research was performed while the second author was visiting MIT, and he thanks the mathematics department for their hospitality. Computations were performed using [Buc99, Ste].

\section{Preliminaries}

We follow the terminology and notation of [Mac95, Sta99] for partitions and tableaux, except where specified. Letting $\mathbb{N}$ denote the nonnegative integers, a partition $\lambda$ of $n \in \mathbb{N}$ is a weakly decreasing sequence $\left(\lambda_{1}, \lambda_{2}, \ldots \lambda_{l}\right)$ of positive integers whose sum is $n$. It will be convenient to set $\lambda_{k}=0$ for $k>l$. We also let $\emptyset$ denote the unique partition with $l=0$. We will identify $\lambda$ with its Young diagram in "French notation": represent the partition $\lambda$ by the unit square cells with top-right corners $(i, j) \in \mathbb{N} \times \mathbb{N}$ such that $1 \leq i \leq \lambda_{j}$. For example, the partition $(4,2,1)$, which we abbreviate as 421 , has Young diagram

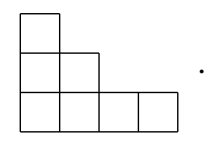


Define the conjugate or transpose $\lambda^{t}$ of $\lambda$ to be the partition with $\lambda_{i}$ cells in column $i$. For example, $421^{t}=3211$. For another partition $\mu$, we write $\mu \subseteq \lambda$ whenever $\mu$ is contained within $\lambda$ (as Young diagrams); equivalently $\mu_{i} \leq \lambda_{i}$ for all $i$. In this case, we define the skew shape $\lambda / \mu$ to be the set theoretic difference $\lambda-\mu$. In particular, the partition $\lambda$ is the skew shape $\lambda / \emptyset$. We call the number of cells of $\lambda / \mu$ its size, denoted $|\lambda / \mu|$. We say that a skew shape forms a horizontal strip (respectively vertical strip) if it contains no two cells in the same column (resp. row). A $k$-horizontal strip is a horizontal strip of size $k$, and similarly for vertical strips. For example, the skew shape $421 / 21$ is a 4-horizontal strip:

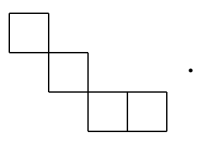

With another skew shape $\sigma / \tau$, we let $(\lambda / \mu) *(\sigma / \tau)$ denote the skew shape obtained by positioning $\lambda / \mu$ so that its bottom right cell is immediately above and left of the top left cell of $\sigma / \tau$. For example, the horizontal strip $421 / 21$ above could alternatively be written as $(21 / 1) *(2)$ or as $(1) *(31 / 1)$.

A Young tableau of shape $\lambda / \mu$ is a map from the cells of $\lambda / \mu$ to the positive integers. A semistandard Young tableau (SSYT) is such a filling which is weakly increasing from left-to-right along each row and strictly increasing up each column, such as

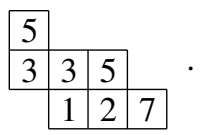

The content of an SSYT $T$ is the sequence $\pi$ such that $T$ has $\pi_{i}$ cells with entry $i$; in this case $\pi=$ $(1,1,2,0,2,0,1)$.

We let $\Lambda$ denote the ring of symmetric functions in the variables $x=\left(x_{1}, x_{2}, \ldots\right)$ over $\mathbb{Q}$, say. We will use three familiar bases from [Mac95, Sta99] for $\Lambda$ : the elementary symmetric functions $e_{\lambda}$, the complete homogeneous symmetric functions $h_{\lambda}$ and, most importantly, the Schur functions $s_{\lambda}$. The Schur functions form an orthonormal basis for $\Lambda$ with respect to the Hall inner product and may be defined in terms of SSYTs by

$$
s_{\lambda}=\sum_{T \in \operatorname{SSYT}(\lambda)} x^{T}
$$

where the sum is over all SSYTs of shape $\lambda$ and where $x^{T}$ denotes the monomial $x_{1}^{\pi_{1}} x_{2}^{\pi_{2}} \cdots$ when $T$ has content $\pi$. Replacing $\lambda$ by $\lambda / \mu$ in 2.1) gives the definition of the skew Schur function $s_{\lambda / \mu}$, where the sum is now over all SSYTs of shape $\lambda / \mu$. For example, the SSYT shown above contributes the monomial $x_{1} x_{2} x_{3}^{2} x_{5}^{2} x_{7}$ to $s_{431 / 1}$.

\section{The skew Pieri rule}

The celebrated Pieri rule gives an elegant method for expanding the product $s_{\lambda} s_{n}$ in the Schur basis. This rule was originally stated in [Pie93] in the setting of Schubert Calculus. Recall that the single row Schur function $s_{n}$ equals the complete homogeneous symmetric function $h_{n}$. Recall also the involution $\omega$ on $\Lambda$, which may be defined by sending the Schur function $s_{\lambda}$ to $s_{\lambda^{t}}$ or equivalently by sending $h_{k}$ to $e_{k}$. Thus the Schur function $s_{1^{n}}$ equals the elementary symmetric function $e_{n}$, where $1^{n}$ denotes a single column of size $n$. 


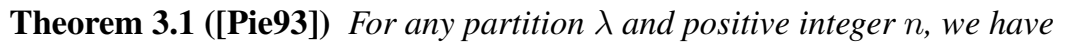

$$
s_{\lambda} s_{n}=s_{\lambda} h_{n}=\sum_{\lambda^{+} / \lambda} s_{n \text {-hor. strip }},
$$

where the sum is over all partitions $\lambda^{+}$such that $\lambda^{+} / \lambda$ is a horizontal strip of size $n$.

Applying the involution $\omega$ to 3.1, we get the dual version of the Pieri rule:

$$
s_{\lambda} s_{1^{n}}=s_{\lambda} e_{n}=\sum_{\lambda^{+} / \lambda} s_{n \text {-vert. strip }} s_{\lambda^{+}},
$$

where the sum is now over all partitions $\lambda^{+}$such that $\lambda^{+} / \lambda$ is a vertical strip of size $n$.

A simple application of Theorem 3.1 gives

$$
s_{322} s_{2}=s_{3222}+s_{3321}+s_{4221}+s_{432}+s_{522},
$$

as represented diagrammatically in Figure 1

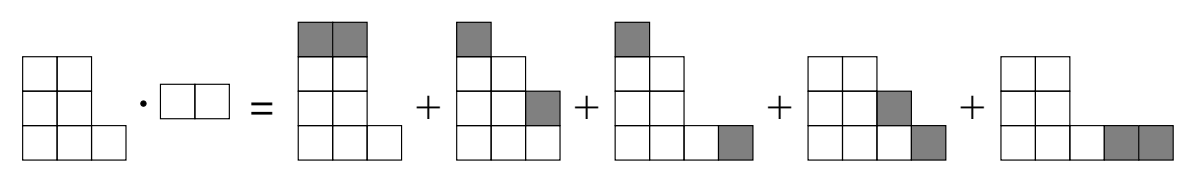

Fig. 1: The expansion of $s_{322} s_{2}$ by the Pieri rule.

Given the simplicity of 3.1], it is natural to hope for a simple expression for $s_{\lambda / \mu} s_{n}$ in terms of skew Schur functions. This brings us to our main result.

Theorem 3.2 For any skew shape $\lambda / \mu$ and positive integer $n$, we have

$$
s_{\lambda / \mu} s_{n}=s_{\lambda / \mu} h_{n}=\sum_{k=0}^{n}(-1)^{k} \sum_{\substack{\lambda^{+} / \lambda \\ \mu / \mu^{-} \\ k \text {-vert. strip }}} s_{\lambda^{+} / \mu^{-}},
$$

where the second sum is over all partitions $\lambda^{+}$and $\mu^{-}$such that $\lambda^{+} / \lambda$ is a horizontal strip of size $n-k$ and $\mu / \mu^{-}$is a vertical strip of size $k$.

Observe that when $\mu=\emptyset$, Theorem 3.2 specializes to Theorem 3.1. Again, we can apply the $\omega$ transformation to obtain the dual version of the skew Pieri rule.

Corollary 3.3 For any skew shape $\lambda / \mu$ and any positive integer $k$, we have

$$
s_{\lambda / \mu} s_{1^{n}}=s_{\lambda / \mu} e_{n}=\sum_{k=0}^{n}(-1)^{k} \sum_{\substack{\lambda^{+} / \lambda(n-k) \text {-vert. strip } \\ \mu / \mu^{-}} \text {-hor. strip }} s_{\lambda^{+} / \mu^{-}},
$$

where the sum is over all partitions $\lambda^{+}$and $\mu^{-}$such that $\lambda^{+} / \lambda$ is a vertical strip of size $n-k$ and $\mu / \mu^{-}$ is a horizontal strip of size $k$. 
Example 3.4 A direct application of Theorem 3.2 gives

$$
\begin{gathered}
s_{322 / 11} s_{2}=s_{3222 / 11}+s_{3321 / 11}+s_{4221 / 11}+s_{432 / 11}+s_{522 / 11} \\
-s_{3221 / 1}-s_{332 / 1}-s_{422 / 1}+s_{322},
\end{gathered}
$$

as represented diagrammatically in Figure 2 .

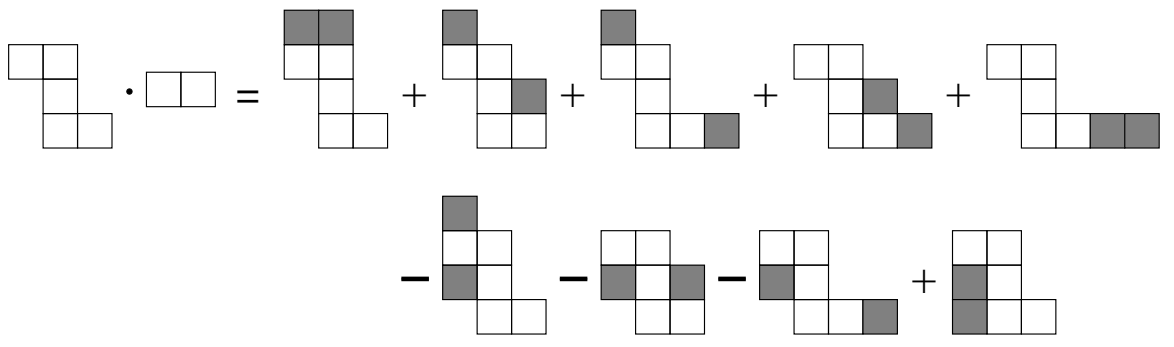

Fig. 2: The expansion of $s_{322 / 11} s_{2}$ by the skew Pieri rule.

As 3.3 contains negative signs, our approach to proving Theorem 3.2 will be to construct a signreversing involution on SSYTs of shapes of the form $\lambda^{+} / \mu^{-}$. We will then provide a bijection between SSYTs of shape $\lambda^{+} / \mu^{-}$that are fixed under the involution and SSYTs of shape $(\lambda / \mu) *(n)$. The result then follows from the fact that $s_{(\lambda / \mu) *(n)}=s_{\lambda / \mu} s_{n}$.

\section{Row insertion}

In order to describe our sign-reversing involution, we will need the Robinson-Schensted-Knuth (RSK) row insertion algorithm on SSYTs [Rob38, Sch61, Knu70]. For a thorough treatment of this algorithm along with many applications, we recommend [Sta99]. In fact, we will use an analogue of the algorithm for SSYTs of skew shape from [SS90]. There, row insertion comes in two forms, external and internal row insertion. External row insertion, which we now define, is just like the classical RSK insertion.

Definition 4.1 Let $T$ be an SSYT of arbitrary skew shape and choose a positive integer $k$. Define the external row insertion of $k$ into $T$, denoted $T \leftarrow_{0} k$, as follows: if $k$ is weakly larger than all entries in row 1 of $T$, then add $k$ to the right end of the row and terminate the process. Otherwise, find the leftmost cell in row 1 of $T$ whose entry, say $k^{\prime}$, is greater than $k$. Replace this entry by $k$ and then row insert $k^{\prime}$ into $T$ at row 2 using the procedure just described. Repeat the process until some entry comes to rest at the right end of a row.

Example 4.2 Let $\lambda / \mu=7541 / 32$ and $\lambda^{+} / \mu^{-}=7542 / 31$ so that the outlined entries below are those in $\lambda / \mu$. The result of externally row inserting $a 2$ is shown below, with changed cells circled.

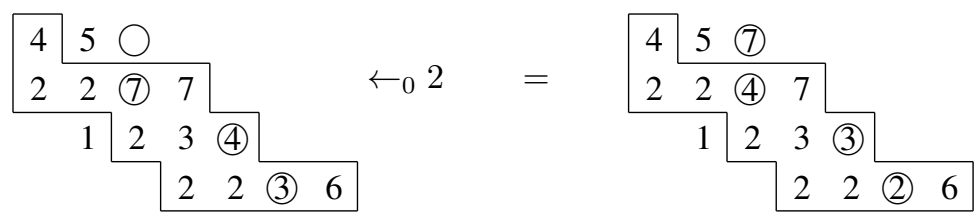


An inside corner (resp. outside corner) of an SSYT $T$ is a cell that has no cell of $T$ immediately below or to its left (resp. above or to its right). Therefore, inside and outside corners are those individual cells whose removal from $T$ still yields an SSYT of skew shape.

Definition 4.3 Let $T$ be an SSYT of arbitrary skew shape and let $T$ have an inside corner in row $r$ with entry $k$. Define the internal row insertion of $k$ from row $r$ into $T$, denoted $T \leftarrow_{r} k$, as the removal of $k$ from row $r$ and its insertion, using the rules for external row insertion, into row $r+1$. The insertion proceeds until some entry comes to rest at the right end of a row.

We could regard external insertions as internal insertions from row 0, explaining our notation. We will simply write $T \leftarrow k$ when specifying the type or row of the insertion is unnecessarily cumbersome.

Example 4.4 Taking $T$ as the SSYT on the right in (4.1), the result of internally row inserting the 1 from row 2 is shown below.

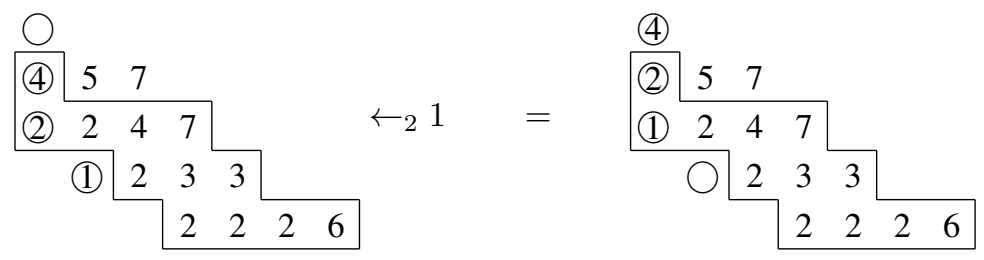

For both types of insertion, we must be a little careful when inserting an entry into an empty row, say row $i$ : in this case $\lambda_{i}=\mu_{i}$ and the entry must be placed in column $\lambda_{i}+1$.

Note that an internal insertion results in the same multiset of entries while an external insertion adds an entry. It is not difficult to check that either operation results in an SSYT.

We will also need to invert row insertions, again for skew shapes and following [SS90].

Definition 4.5 Let $T$ be an SSYT of arbitrary skew shape and choose an outside corner c of $T$, say with entry $k$. Define the reverse row insertion of $c$ from $T$, denoted $T \rightarrow c$, by deleting $c$ from $T$ and reverse inserting $k$ into the row below, say row $r$, as follows: if $r=0$, then the procedure terminates. Otherwise, if $k$ is weakly smaller than all entries in row $r$, then place $k$ at the left end of row $r$ and terminate the procedure. Otherwise, find the rightmost cell in row $r$ whose entry, say $k^{\prime}$, is less than $k$. Replace this entry by $k$ and then reverse row insert $k^{\prime}$ into row $r-1$ using the procedure just described.

Example 4.6 In (4.1), reverse row insertion of the cell containing the circled 7 from the SSYT on the right results in the SSYT on the left, and similarly in 4.2) for the circled 4.

As with row insertion, it follows from the definition that the resulting array will again be an SSYT. Observe that the first type of termination mentioned in Definition 4.5 corresponds to reverse external row insertion, and we then say that $k$ lands in row 0 . The second type of termination corresponds to reverse internal row insertion, and we then say that $k$ lands in row $r$. In both cases, we will call the entry $k$ left at the end of the procedure the final entry of $T \rightarrow c$. The following lemma, which follows immediately from Definitions 4.1, 4.3 and 4.5, formalizes the bijectivity of row and reverse row insertion.

Lemma 4.7 Let $T$ be an SSYT of skew shape.

a. If $S$ is the result of $T \leftarrow k$ for some positive integer $k$, then $S \rightarrow c$ results in $T$, where $c$ is the unique non-empty cell of $S$ that is empty in $T$. 
b. If $S$ is the result of $T \rightarrow c$ for some removable cell $c$ of $T$ and the final entry $k$ of $T \rightarrow c$ lands in row $r \geq 0$, then $S \leftarrow_{r} k$ results in $T$.

For both row insertion and reverse row insertion, we will often want to track the cells affected by the procedure. Therefore define the bumping path of the row insertion $T \leftarrow k$ (resp. the reverse bumping path of a reverse row insertion $T \rightarrow c$ ) to be the set of cells in $T$, as well as those empty cells, where the entries differ from the corresponding entries in $T \leftarrow k$ (resp. $T \rightarrow c$ ). The cells of the bumping paths for row insertion and reverse row insertion are circled in (4.1) and (4.2). Note that the fact that the bumping path and reverse bumping path are equal in each of these examples is a consequence of Lemma 4.7 .

It is easy to see that the bumping paths always move weakly right from top to bottom in the case of column-strict tableaux. The following bumping lemma will play a crucial role in defining our signreversing involution and in proving its relevant properties.

Lemma 4.8 Let $T$ be an SSYT of skew shape and let $k, k^{\prime}$ be positive integers. Let $B$ be the bumping path of $T \leftarrow k$ and let $B^{\prime}$ be the bumping path of $(T \leftarrow k) \leftarrow k^{\prime}$.

a. If $B$ is strictly left of $B^{\prime}$ in any row $r$, then $B$ is strictly left of $B^{\prime}$ in every row they both occupy. Moreover, the top cells of $B$ and $B^{\prime}$ form a horizontal strip.

b. If both row insertions are external, then $B$ is strictly left of $B^{\prime}$ in every row they both occupy if and only if $k \leq k^{\prime}$.

c. Suppose $C^{\prime}$ is the reverse bumping path of $T \rightarrow c^{\prime}$ with final entry $k^{\prime}$ and $C$ is the reverse bumping path of $\left(T \rightarrow c^{\prime}\right) \rightarrow c$ with final entry $k$. If $c$ is strictly left of $c^{\prime}$, then $C$ is strictly left of $C^{\prime}$ in every row they both occupy. If, in addition, both reverse row insertions land in row 0 , then $k \leq k^{\prime}$.

To foreshadow the role of Lemma 4.8 in the following section, we give a proof of the classical Pieri rule using this result.

Proof of Theorem 3.1: The formula is proved if we can give a bijection between SSYTs of shape $\lambda *(n)$ and SSYTs of shape $\lambda^{+}$such that $\lambda^{+} / \lambda$ is a horizontal strip of size $n$. Let $k_{1} \leq \cdots \leq k_{n}$ be entries of $(n)$ from left to right. Repeated applications of (a) and (b) of Lemma 4.8 ensure that row inserting these entries into an SSYT of shape $\lambda$ will add a horizontal strip of size $n$ to $\lambda$. By Lemma 4.7, this establishes a bijection where the inverse map is given by reverse row inserting the cells of $\lambda^{+} / \lambda$ from right to left.

\section{A sign-reversing involution}

Throughout this section, fix a skew shape $\lambda / \mu$. We will be interested in SSYTs of shape $\lambda^{+} / \mu^{-}$, where we always assume that $\lambda^{+} / \lambda$ is a horizontal strip, $\mu / \mu^{-}$is a vertical strip, and $\left|\lambda^{+} / \lambda\right|+\left|\mu / \mu^{-}\right|=n$. Our goal is to construct a sign-reversing involution on SSYTs whose shapes take the form $\lambda^{+} / \mu^{-}$, such that the fixed points are in bijection with SSYTs of shape $(\lambda / \mu) *(n)$.

Our involution is reminiscent of the proof of the classical Pieri rule given in Section 4 . By Lemma 4.7. reverse row insertion gives a bijective correspondence provided we record the final entry and its landing row. Our strategy, then, is to reverse row insert the cells of $\lambda^{+} / \lambda$ from right to left, recording the entries as we go. If at some stage we land in row $r \geq 1$, we will then re-insert all the previous final entries. More formally, we have the following definition of a downward slide of $T$. 
Definition 5.1 Let $T$ be an SSYT of shape $\lambda^{+} / \mu^{-}$. Define the downward slide of $T$, denoted $\mathrm{D}(T)$, as follows: construct $T \rightarrow c_{1}$ where $c_{1}$ is the rightmost cell of $\lambda^{+} / \lambda$, and let $k_{1}$ denote the final entry. If $k_{1}$ lands in row 0 , then continue with $c_{2}$ the second rightmost cell of $\lambda^{+} / \lambda$ and $k_{2}$ the final entry of $\left(T \rightarrow c_{1}\right) \rightarrow c_{2}$. Continue until the first time $k_{m}$ lands in row $r \geq 1$ and set $m^{\prime}=m-1$, or set $m=m^{\prime}=\left|\lambda^{+} / \lambda\right|$ if no such $k_{m}$ exists. Then $\mathrm{D}(T)$ is given by

$$
\left(\cdots\left(\left(\left(\cdots\left(T \rightarrow c_{1}\right) \rightarrow c_{2} \cdots\right) \rightarrow c_{m}\right) \leftarrow k_{m^{\prime}}\right) \cdots\right) \leftarrow k_{1} .
$$

Example 5.2 With $T$ shown on the left below, we exhibit the construction of $\mathrm{D}(T)$ in two steps. We find that $m=4$ and the middle SSYT shows the result of $\left(\left(\left(T \rightarrow c_{1}\right) \rightarrow c_{2}\right) \rightarrow c_{3}\right) \rightarrow c_{4}$. The entries that land in row 0 are recorded in the dashed box. Then the SSYT on the right is $\mathrm{D}(T)$. The significance of the circles will be explained later.
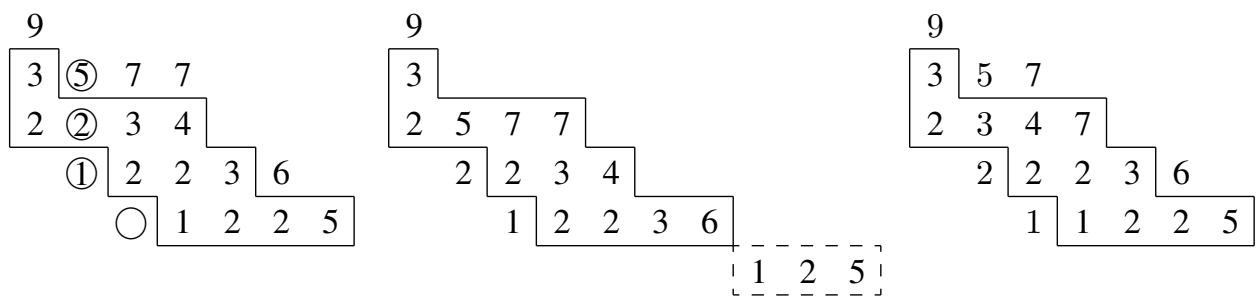

Alternatively, if $T$ is the SSYT shown on the left below, we find that $m=3$ and that all three final entries land in row 0. Then $\mathrm{m}^{\prime}=\left|\lambda^{+} / \lambda\right|$ and Lemma 4.7 ensures that $D(T)=T$. Below in the middle, we have shown $\left(\left(T \rightarrow c_{1}\right) \rightarrow c_{2}\right) \rightarrow c_{3}$. The position of the dashed box is intended to be suggestive: together with the entries in the outlined shape, we see that we have an SSYT of shape $(\lambda / \mu) *(n)=(653 / 21) *(3)$.
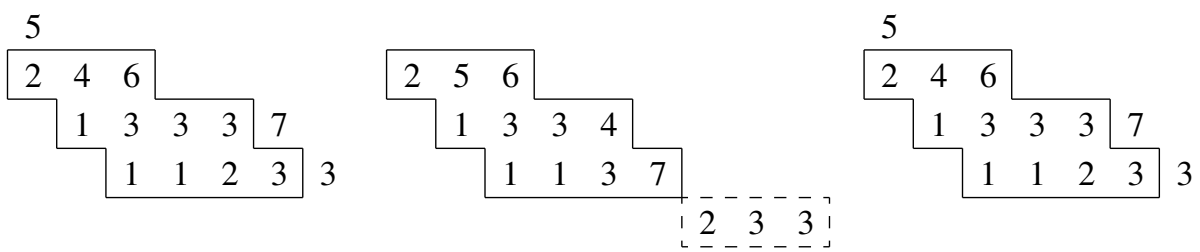

The final reverse bumping path in a downward slide will play an important role in the sign-reversing involution. Therefore, with notation as in Definition 5.1 if $m<\left|\lambda^{+} / \lambda\right|$, then we refer to the reverse bumping path of $T \rightarrow c_{m}$ as the downward path of $T$. The cells of the downward path of $T$ are circled above. Say that the downward path of $T$ exits right if its bottom cell (which may be empty) is strictly below the bottom cell $\mu / \mu^{-}$. Our terminology is justified since one can show that the exits right condition is equivalent to the bottom cell of the downward path being weakly right of the bottom cell of $\mu / \mu^{-}$. The importance of the exits right condition is revealed by the following result.

Proposition 5.3 Suppose $T$ is an SSYT of shape $\lambda^{+} / \mu^{-}$such that the downward path of $T$, if it exists, exits right. Then $\mathrm{D}(T)$ is an SSYT of shape $\lambda^{\prime} / \mu^{\prime}$, where $\lambda^{\prime} / \lambda\left(\right.$ resp. $\left.\mu / \mu^{\prime}\right)$ is a horizontal (resp. vertical) strip. 
Supposing $\mathrm{D}(S)=T$ with $T \neq S$, the next step is to invert the downward slides for such $T$. Since any such $T$ necessarily has $\mu^{-} \neq \mu$, the idea is to internally row insert the bottom cell of $\mu / \mu^{-}$. However, before doing this we must reverse row insert certain cells of $\lambda^{+} / \lambda$, as in a downward slide. To describe which cells to reverse insert, we define the upward path of $T$ to be the bumping path that would result from internal row insertion of the entry in the bottom cell of $\mu / \mu^{-}$. Roughly, we will reverse row insert anything that is weakly right of this upward path.

Definition 5.4 Let $T$ be an SSYT of shape $\lambda^{+} / \mu^{-}$such that $\mu^{-} \neq \mu$. Define the upward slide of $T$, denoted $\mathrm{U}(T)$, as follows: construct $T \rightarrow c_{1}$ where $c_{1}$ is the rightmost cell of $\lambda^{+} / \lambda$, and let $k_{1}$ denote its final entry and $B_{1}$ its bumping path. If $B_{1}$ fails to stay weakly right of the upward path of $T$, then set $m=m^{\prime}=0$. Otherwise, consider $c_{2}$, the second rightmost cell of $\lambda^{+} / \lambda$, and $k_{2}$, the final entry of $\left(T \rightarrow c_{1}\right) \rightarrow c_{2}$, and $B_{2}$, the corresponding bumping path. Continue until the last time $B_{m}$ stays weakly right of the upward path of $T$ or until no cell of $\lambda^{+} / \lambda$ remains. Suppose that after the reverse row insertions, the bottom cell of $\mu / \mu^{-}$is in row $r$ and has entry $k$. Then $\mathrm{U}(T)$ is given by

$$
\left(\cdots\left(\left(\left(\left(\cdots\left(T \rightarrow c_{1}\right) \rightarrow c_{2} \cdots\right) \rightarrow c_{m}\right) \leftarrow_{r} k\right) \leftarrow k_{m^{\prime}}\right) \cdots\right) \leftarrow k_{1}
$$

where we set $m^{\prime}=m$ if $k_{m}$ lands in row 0 , and $m^{\prime}=m-1$ otherwise.

Example 5.5 Letting $T$ be the rightmost SSYT of (5.1), the cells of the upward path of $T$ are circled below. We determine $\mathrm{U}(T)$ in three steps. We find that $m=3$ and the middle SSYT of (5.1) shows $\left(\left(T \rightarrow c_{1}\right) \rightarrow c_{2}\right) \rightarrow c_{3}$. Then $\left(\left(\left(T \rightarrow c_{1}\right) \rightarrow c_{2}\right) \rightarrow c_{3}\right) \leftarrow k$ is shown in the middle below, while $\mathrm{U}(T)$ is shown on the right. Comparing with Example 5.2. we observe that the upward slide in this case does indeed invert the downward slide.
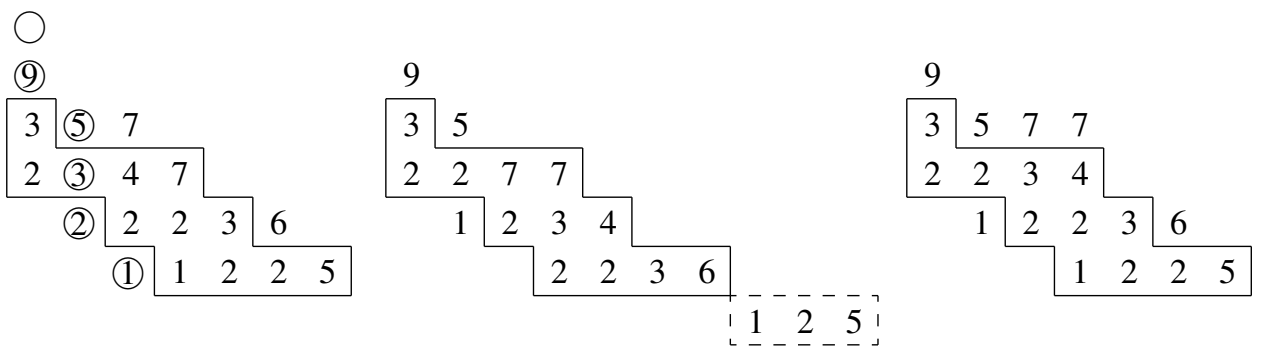

There are also instances where the entry $k$ of Definition 5.4 is different from the entry originally at the bottom of the upward path. For example, the same three-step process for constructing $\mathrm{U}(T)$ is shown below for an example with $m=2$. There, $k=2$, even though the original upward path of $T$ had 1 as its bottom entry.

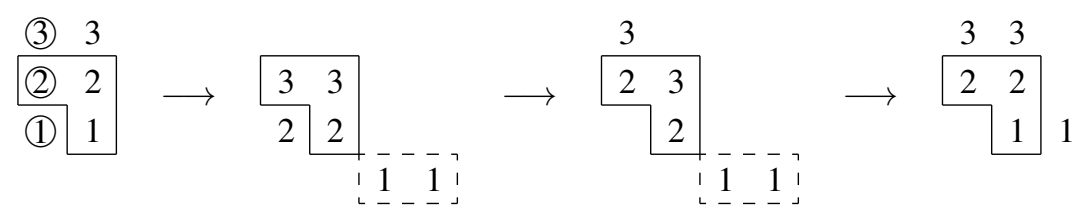


As with downward slides and before presenting our involution, we must ensure that the result of an upward slide is always a tableau of the appropriate skew shape.

Proposition 5.6 Suppose T is an SSYT of shape $\lambda^{+} / \mu^{-}$such that in the upward slide of T, all the final entries of the reverse row insertions land in row 0 . Then $\mathrm{U}(T)$ is an SSYT of shape $\lambda^{\prime} / \mu^{\prime}$, where $\lambda^{\prime} / \lambda$ (resp. $\left.\mu / \mu^{\prime}\right)$ is a horizontal (resp. vertical) strip.

Our involution will consist of either applying a downward slide or an upward slide. The decision for which slide to apply is roughly based on which of the downward path of $T$ or the upward path of $T$ lies further to the right.

Definition 5.7 Consider the set of SSYTs T of shape $\lambda^{+} / \mu^{-}$such that that $\lambda^{+} / \lambda$ is a horizontal strip and $\mu / \mu^{-}$is a vertical strip. Define a map $\phi$ on such $T$ by

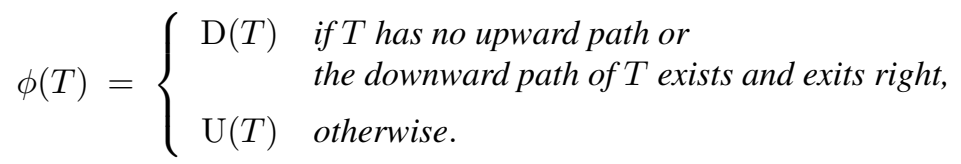

Theorem 5.8 The map $\phi$ defines an involution on the set of SSYTs with shapes of the form $\lambda^{+} / \mu^{-}$where $\lambda^{+} / \lambda$ is a horizontal strip and $\mu / \mu^{-}$is a vertical strip.

We now have all the ingredients needed to prove the skew Pieri rule.

Proof of Theorem 3.2; Using the expansion of $s_{\lambda^{+}} / \mu^{-}$in terms of SSYTs as in (2.1), observe that if $\phi(T) \neq T$, then the $T$ and $\phi(T)$ occur with different signs in the right-hand side of 3.3 . Since $\phi$ clearly preserves the monomial associated to an SSYT, the monomials corresponding to $T$ and $\phi(T)$ in the right-hand side of (3.3) will cancel out. Because $s_{\lambda / \mu} s_{n}=s_{(\lambda / \mu) *(n)}$, it remains to show that there is a monomial-preserving bijection from fixed points of $\phi$ to SSYTs of shape $(\lambda / \mu) *(n)$.

Note that $T$ is a fixed point of $\phi$ only if $T$ has neither an upward path nor a downward path. This happens if and only if $\mu^{-}=\mu$ and when reverse row inserting the cells of $\lambda^{+} / \lambda$ from right to left, every final entry lands in row 0 . In particular, the entries of $T$ remaining after reverse row inserting the cells of $\lambda^{+} / \lambda$ form an SSYT of shape $\lambda / \mu$. Say the final entries of the reverse row insertions are $k_{n}, \ldots, k_{1}$ in the order removed. By Lemma 4.8 (c), since $\lambda^{+} / \lambda$ is a horizontal strip, we have $k_{1} \leq \cdots \leq k_{n}$ and so these entries form an SSYT of shape $(n)$. By Lemma 4.7 , this process is invertible and therefore establishes the desired bijection.

Remark 5.9 We proved Theorem 3.2 by working with SSYTs. In particular, we showed that the two sides of (3.3) were equal when expanded in terms of monomials. Alternatively, we could consider the expansions of both sides of (3.3) in terms of Schur functions. The Littlewood-Richardson rule states that the coefficient of $s_{\nu}$ in the expansion of any skew Schur function $s_{\lambda / \mu}$ is the number of LittlewoodRichardson fillings (LR-fillings) of shape $\lambda / \mu$ and content $\nu$. (The interested reader unfamiliar with LR-fillings can find the definition in [Sta99].) It is not hard to check that our maps $\mathrm{D}$ and $\mathrm{U}$ send LRfillings to LR-fillings, and bumping within LR-fillings has some nice properties. For example, the entries along a (reverse) bumping path are always $1,2, \ldots, r$ from bottom to top for some $r$.

However, we chose to give our proof in terms of SSYTs because one does not need to invoke the power of the Littlewood-Richardson rule to prove the classical Pieri rule, and we wanted the same to apply to the skew Pieri rule. 


\section{References}

[AM09] Sami H. Assaf and Peter R. W. McNamara. A Pieri rule for skew shapes. Preprint. With an appendix by Thomas Lam. arXiv:0908.0345, 2009.

[Buc99] Anders S. Buch. Littlewood-Richardson calculator, 1999. Available from http://www. math.rutgers.edu/ asbuch/lrcalc.

[Knu70] Donald E. Knuth. Permutations, matrices, and generalized Young tableaux. Pacific J. Math., 34:709-727, 1970.

[Koo88] Tom H. Koornwinder. Self-duality for $q$-ultraspherical polynomials associated with root system $a_{n}$. Unpublished manuscript. http://staff.science.uva.nl/ thk/art/ informal/dualmacdonald.pdf, 1988.

[Lam05] Thomas Lam. Ribbon tableaux and the Heisenberg algebra. Math. Z., 250(3):685-710, 2005.

[Las89] Michel Lassalle. Une formule de Pieri pour les polynômes de Jack. C. R. Acad. Sci. Paris Sér. I Math., 309(18):941-944, 1989.

[LLS09] Thomas Lam, Aaron Lauve, and Frank Sottile. Skew Littlewood-Richardson rules from Hopf algebras. Preprint. arXiv:0908.3714, 2009.

[LR34] D.E. Littlewood and A.R. Richardson. Group characters and algebra. Philos. Trans. Roy. Soc. London, Ser. A, 233:99-141, 1934.

[LS82] Alain Lascoux and Marcel-Paul Schützenberger. Polynômes de Schubert. C. R. Acad. Sci. Paris Sér. I Math., 294(13):447-450, 1982.

[LS07] Cristian Lenart and Frank Sottile. A Pieri-type formula for the $K$-theory of a flag manifold. Trans. Amer. Math. Soc., 359(5):2317-2342 (electronic), 2007.

[Mac87] I. G. Macdonald. The symmetric functions $P(x ; q, t)$ : facts and conjectures. Unpublished manuscript, 1987.

[Mac95] I. G. Macdonald. Symmetric functions and Hall polynomials. Oxford Mathematical Monographs. The Clarendon Press Oxford University Press, New York, second edition, 1995. With contributions by A. Zelevinsky, Oxford Science Publications.

[Man98] Laurent Manivel. Fonctions symétriques, polynômes de Schubert et lieux de dégénérescence, volume 3 of Cours Spécialisés [Specialized Courses]. Société Mathématique de France, Paris, 1998.

[Mor64] A. O. Morris. A note on the multiplication of Hall functions. J. London Math. Soc., 39:481-488, 1964.

[Pie93] Mario Pieri. Sul problema degli spazi secanti. Rend. Ist. Lombardo (2), 26:534-546, 1893.

[Rob38] G. de B. Robinson. On the Representations of the Symmetric Group. Amer. J. Math., 60(3):745$760,1938$. 
[Sch61] C. Schensted. Longest increasing and decreasing subsequences. Canad. J. Math., 13:179-191, 1961.

[Sch77] M.-P. Schützenberger. La correspondance de Robinson. In Combinatoire et représentation du groupe symétrique (Actes Table Ronde CNRS, Univ. Louis-Pasteur Strasbourg, Strasbourg, 1976), pages 59-113. Lecture Notes in Math., Vol. 579. Springer, Berlin, 1977.

[Sot96] Frank Sottile. Pieri's formula for flag manifolds and Schubert polynomials. Ann. Inst. Fourier (Grenoble), 46(1):89-110, 1996.

[SS90] Bruce E. Sagan and Richard P. Stanley. Robinson-Schensted algorithms for skew tableaux. $J$. Combin. Theory Ser. A, 55(2):161-193, 1990.

[Sta89] Richard P. Stanley. Some combinatorial properties of Jack symmetric functions. Adv. Math., 77(1):76-115, 1989.

[Sta99] Richard P. Stanley. Enumerative combinatorics. Vol. 2, volume 62 of Cambridge Studies in Advanced Mathematics. Cambridge University Press, Cambridge, 1999.

[Ste] John R. Stembridge. SF, posets and coxeter/weyl. Available from http://www . math. Isa. umich.edu/ jrs/maple.html.

[Tho74] Glânffrwd P. Thomas. Baxter algebras and Schur functions. PhD thesis, University College of Swansea, 1974.

[Tho78] Glânffrwd P. Thomas. On Schensted's construction and the multiplication of Schur functions. Adv. in Math., 30(1):8-32, 1978.

[Win98] Rudolf Winkel. On the multiplication of Schubert polynomials. Adv. in Appl. Math., 20(1):7397, 1998. 